Artículo

\title{
La comprensión lectora: hacia una aproximación sociocultural
}

\author{
Reading comprehension: a sociocultural approach \\ Luisa Emilia Sánchez Chévez* \\ lemsach@live.com
}

ISSN 1996-1642, Editorial Universidad Don Bosco, año 7, No.12, Julio-Diciembre de 2013, pp.7-16

Recibido: 15 de julio de 2013. Aceptado: 9 de agosto de 2013

Resumen

El presente artículo aborda el tema de la comprensión lectora. Se analiza la evolución de sus conceptos a partir de los estudios de la Psicología Cognitiva y luego se enfatiza en el enfoque sociocultural de la lectura. Finalmente se concluye que entre ambas corrientes ha de establecerse un consenso para lograr una conceptualización más integral sobre el tema de la comprensión lectora.

Palabras clave: comprensión lectora, aproximación sociocultural, lectura práctica social, contexto, autor, lector.

\section{Abstract}

This paper approaches reading comprehension. It analyzes the evolution of reading comprehension concepts, first from the studies of the Cognitive Psychology and second from the sociocultural approach. Finally, it concludes that there is a need to establish a consensus to bridge both trends and build a more solid and integral conceptualization of reading comprehension.

Keywords: reading comprehension, sociocultural approach, reading, social practice, context, author, reader.

\footnotetext{
* Candidata a Doctora en Educación por la Universidad de Costa Rica. Docente y Directora del Departamento de Capacitación Metodológica y Transformación Curricular. Universidad Evangélica Nicaragüense Martin Luther King Jr. Managua, Nicaragua.
} 


\section{Introducción}

La lectura se considera una herramienta fundamental para sobrevivir en nuestra sociedad, pues cada día se produce grandes volúmenes de información escrita, que ha de ser leída e interpretada críticamente por la ciudadanía, para conocer y comprender los diversos puntos de vista e intereses que se ocultan detrás de cada texto (Cassany, 2010). Además, la lectura mejora las relaciones familiares y sociales, estimula la imaginación, nuestros conocimientos y nos acerca a una mejor interpretación del medio en que vivimos.

Por tanto, es necesario acceder a la lectura de manera adecuada para así lograr un grado significativo en el desarrollo de la competencia lectora, y favorecer el crecimiento personal y profesional de tal manera que se logre una buena participación social. Por ello, el desarrollo de la comprensión lectora es una de las tareas primordiales en los diversos niveles escolares, en la que están implicados todos los agentes involucrados en la comunidad educativa.

El cumplimiento de esta tarea demanda de un profesorado con sólidos conocimientos disciplinares y una mirada clara sobre lo que significa la expresión "comprender". En el transcurso del tiempo esta concepción ha logrado algunas modificaciones que han robustecido y ampliado su significado. Al respecto Cassany (2008) afirma que se han realizado importantes investigaciones psicolingüísticas sobre el tema de la comprensión lectora, que estas aportan numerosas teorías sobre lo que ocurre en nuestra mente al comprender un texto. Pero estas teorías no se acercan a una interpretación sociocultural de la lectura, que den cuenta de las características propias de las comunidades de los hablantes, desde sus formas de pensar, sus formas leer, sus formas de comprender y los elementos contextuales que rodean al lector en el momento de leer y apropiarse de la nueva información.

Se pretende entonces, no ignorar que la comprensión de la lectura es un proceso dinámico, de alto nivel, que requiere de todos los sistemas atencionales y de memoria, de los procesos de codificación y percepción, de pensamiento y de lenguaje, a si como un sinfín de operaciones inferenciales, basadas en los conocimientos previos y en factores contextuales (Escudero, 2010). Se trata de ahondar y enriquecer el concepto de compresión lectora, valorando también los datos y teorías de la concepción sociocultural de la lectura y escritura, la cual considera el carácter social tanto de los conocimientos y experiencias del lector como del escritor. En este sentido, sería oportuno aprovechar los estudios realizados por estas teorías y elaborar una práctica educativa integradora (Cassany, 2009). 
Visto de esta manera, una mirada más amplia y profunda sobre la concepción de la comprensión lectora será de mucho interés para todos aquellos que participan en la enseñanza de la lectura. Se considera que un buen entendimiento sobre la comprensión lectora, llevará a mejores prácticas pedagógicas, una mejor interpretación de los significados lingüísticos, y por ende, una visión más amplia de mundo. En este artículo, se aborda el tema de la comprensión lectora, hacia una aproximación sociocultural. Con el fin de lograr una mayor amplitud en su concepto, integrando las diferencias y realidades culturales de cada grupo social.

\section{Aproximación sociocultural de la comprensión lectora}

Como bien se expresa en la introducción de este artículo, el concepto de comprensión lectora se ha venido modificando progresivamente, en base a la evolución de la psicología cognitiva y los nuevos estudios socioculturales de la lectura y escritura. Por tanto, hoy día, no podemos reducir el acto de leer al simple hecho de pasar la vista por las líneas del texto e identificar la información literal que lo compone, sin dar paso a la comprensión, que es el producto esencial de la lectura. Desde una mirada cognitiva, la comprensión implica un conjunto de procesos perceptivos, de codificación y acceso léxico, de procesamiento sintáctico y gramatical, de inferencias, de la construcción semántica del texto (León, 2001; León y Escudero, 2003; 2007; Escudero, 2010). Estos procesos, requieren para su desarrollo, un importante esfuerzo por parte del lector, para conjugar estas actividades y llegar a la comprensión global del texto y ampliar o modificar la visión de mundo que posee (Sánchez, 1993).

Desde una concepción sociocultural también se han realizado importantes estudios sobre el tema de la lectura y escritura (Cassany, 2002; 2006; 2008; 2009; 2010; Ames, 2000, Kalman, 2000; 2002; 2003; Murillo, 2009; Zavala, 2009;). Los resultados de estos estudios dan cuenta de numerosas teorías, relacionadas con la transformación en los modos y prácticas del leer y del escribir, considerando su importancia en la formación del sujeto y su participación social.

Hablar de una aproximación sociocultural de la comprensión lectora, significa interpretarla a partir de una realidad social Cassany y Morales (2008), donde se presentan diversos hechos y situaciones sociales, políticos, económicos y culturales. Estos hechos se describen y se divulgan a través de la escritura, en (periódicos, revistas, libros, novelas etc.). Tener conocimiento pleno de estos movimientos sociales permite a los ciudadanos poder opinar y argumentar sobre ello. Por tanto, se subraya desde una concepción sociocultural que la lectura es práctica social, dado que se lee, se interpreta y comprende los hechos históricos de una sociedad, para luego actuar con lo aprendido, tomar decisiones y dar respuesta adecuadas a problemas del entorno. 
En palabras de Cassany (2006), los aportes de investigaciones relacionados con las destrezas cognitivas de la comprensión, son muy importantes. Pues, nos orientan a tener una visión clara, sobre los procesos mentales que se conjugan para llegar a la comprensión total de un texto. Pero estos aportes, no nos aproxima a los componentes culturales que adopta la lectura en cada contexto social, para tener una concepción más sólida e integral de la comprensión. De acuerdo a estas consideraciones, es preciso repensar la conceptualización de la comprensión lectora, valorando que los seres humanos internalizamos procesos cognitivos experimentados en un determinado contexto social (León, et al. 2011 ) donde predomina un bagaje cultural, unas tradiciones, unas costumbres, unas creencias, unos intereses propios de los hablantes de una comunidad determinada.

Dicho de esta manera, la concepción sociocultural nos acerca a una nueva interpretación de la comprensión de la lectura, la cual, se sustenta en un proceso de interacción, dado en un contexto de mayor amplitud. Donde se considera el aprendizaje de la lectura como una herramienta de gran potencial, que prepara al aprendiz para participar activamente en la sociedad, comprender y explicar los sucesos ocurridos en el medio y lograr una mejor convivencia en la comunidad (Kalman, 2000).

Cassany (2006), en sus estudios distingue tres interpretaciones. Una interpretación de la comprensión desde una mirada lingüística, que supone que el significado está en el texto, independientemente de los conocimientos y experiencias de lectores. Una interpretación desde la psicología cognitiva, la cual considera que en la comprensión de un texto, el lector participa con diversas operaciones mentales y construye el significado.

Finalmente, el autor nos acerca hacia una interpretación sociocultural de la comprensión lectora, la cual enfatiza el carácter social tanto de los conocimientos del lector como de gran parte de los significados del texto. El autor reafirma que cada comunidad usa y conforma los textos de una manera peculiar, dándoles un sentido específico, según sean los intereses de la cultura predominante en el tiempo, de su geografía, de sus historias y formas de interpretación de los hablantes. Los aportes de Kalman (2003) y Zavala (2009) expresan que uno de los hallazgos más relevantes en investigaciones reciente, son las diferentes formas de usar la lectura y escritura, pues estas prácticas se sujetan a las particularidades del contexto de su uso, a los objetivos del lector, a la importancia que este da al acto de leer y formas de comprender.

La comprensión lectora:

hacia una aproximación 10. sociocultural
En este sentido se puede sintetizar que los estudios socioculturales se fundamentan en la interpretación y explicación de los elementos contextuales 
relacionados con el ejercicio de la lectura, tomando en cuenta los procesos de aprendizaje, las posibilidades de acceder a la lectura, la forma en que esta se orienta en cada centro de estudio y su relación con la organización social. Desde una mirada sociocultural, la comprensión de la lectura ha de ser entendida entonces, como un proceso eminentemente social, ya que, según lo indica Murillo (2009), los textos orientados para lectura son discursos que responden a diversos patrones culturales, presentes en una sociedad. Estos varían de acuerdo a la época, a la historia, a los avances tecnológicos, a las formas de gobierno y políticas económicas presentes en el momento. Por tanto, indica Cassany (2009) se precisa de unas buenas prácticas de lectura, orientadas a la interpretación y comprensión de la ideología presente en cada texto, para enriquecer la ideología propia y participar en la sociedad con un actitud sólida y propositiva para el bien de la nación.

\section{La lectura como práctica cultural}

Las prácticas culturales las define Contreras (2008) como las actividades específicas que realizan los integrantes de una comunidad determinada -artísticas, académicas, religiosas, deportivas, escolares, científicas, etcétera--. Estos espacios sociales se van consolidando a lo largo de la historia y se orientan a la formación de una identidad, que es a la vez, una formación personal y social. Situándonos en la práctica de la lectura, esta es concebida como una práctica sociocultural, puesto que da origen a interpretaciones e intercambios sociales y parte de las condiciones propias del autor y su mundo inmerso en el texto, más el bagaje cultural que forma parte de la formación del lector. La lectura se visualiza como una práctica en el momento en que el lector es capaz de crecer personalmente, ubicarse en un determinado ámbito empresarial, sostener relaciones comunicativas con sus compañeros, utiliza el aprendizaje para gestionar actividades personales, laborales y ayudar a los otros cada vez que se requiera.

En el sentido específico de la lectura (Cassany y Morales, 2008), afirman que leer y escribir son acciones culturales, presentes en un contexto social, y que estas varían de acuerdo a las características de los hablantes, de su forma de expresión, interpretación y participación en la sociedad. Con esta misma ideología Kalman (2000) sostiene que la lectura se contextualiza en cada espacio social y nos orienta a una conexión con el mundo actual.

En efecto, cada sociedad, cada país, cada región refleja en su interior mundos distintos. Desde su ubicación geográfica, diversas formas de organización, de producción, de interpretar la información, los objetos y las cosas. La escritura no es ajena a estos elementos contextuales, por el contrarios se sustenta en ellos, ya que el autor estructura la información de un texto en base a sus vivencias, 
experiencias y expresiones propias en un espacio social determinado. "Los discursos son textos en contextos que tienen una función comunicativa en una determinada sociedad" (Murillo, 2009, p. 12). Es así que la lectura nos aproxima a otras culturas y miradas de mundo, fortalece el pensamiento y amplia el universo de oportunidades en la sociedad.

Siguiendo a Cassany (2006), uno de los desafíos que enfrenta el estudiantado que entra por primera vez a la universidad es el aprendizaje de las nuevas prácticas letradas, según sea la disciplina de estudio, pues éstas se caracterizan por contener discursos un tanto complejos y requieren de un alto nivel de comprensión para construir el significado. Asimismo, continúa Carlino (2005), para el éxito del aprendizaje en la universidad, es fundamental fomentar la interacción entre los estudiantes, docentes e instituciones educativas. Pues, los estudiantes requieren para su formación profesional, que se ventile desde las aulas de clase una buena comunicación entre compañeros, para garantizar así, la movilidad de conocimientos y formas culturales.

Además, indica Aliagas (s. f.) es fundamental que se oriente desde el currículo escolar, la creación de espacios de intercambio. Estos espacios se vuelven muy productivos en la medida que los estudiantes puedan compartir sus experiencias y realizar actividades de aprendizajes que sean de su interés. En este aspecto, los docentes han de saber brindar las ayudas oportunas y pertinentes, en base a las diferencias y particularidades de cada estudiante, para lograr el intercambio ideológico entre aprendices y docentes. Además de establecer las conexiones precisas entre padres de familia y personas involucradas en el ámbito educativo para consensuar las mejores formas de orientar las prácticas letradas a los estudiantes, respetando los aspectos tradicionales propios de cada ser.

\section{La lectura como práctica social}

Gran parte de la actividad escolar está organizada en torno a la comunicación escrita. Los estudiantes han de leer, comprender, valorar críticamente la información contenida en los textos y almacenarla en su memoria, de modo que puedan usarla posteriormente con facilidad. En las aulas de clase los docentes fomentan las discusiones de grupo, los estudiantes participan, argumentan, dan sus puntos de vistas sobre lo comprendido del texto, mostrando a si, la utilidad de comprender la información. En este sentido, para Cassany (2009), comprender es una práctica social que se nutre con el pensamiento del otro, requiere de las ayudas de otros, de los aportes de otros, de las vivencias y saberes de otros para llegar a un entendimiento más integral de la información. Igualmente los aportes de Kalman (2003) indican que la interacción con otros, orienta al

La comprensión lectora:

hacia una aproximación

12. sociocultural individuo hacia las prácticas sociales, en la medida en que el conocimiento que se adquiere de manera individual es también un producto social, pues se 
obtiene de la interpretación de diversas fuentes ideológicas y valores culturales que subyacen en cada escrito.

De tal manera que podemos concluir que el éxito de la lectura es la comprensión. Mas, comprender significa, desde una concepción sociocultural, usar de manera adecuada la lengua escrita, identificar los géneros textuales, los discursos, los significados y apropiarse de los aspectos culturales presentes en cada texto, para poder participar con solidez y confianza en diversos eventos sociales, y tener un comportamiento humano de calidad. Finalmente se subraya que la comprensión lectora es una actividad eminentemente social, que se sustenta en la interpretación de diversos mundos revelados por el escritor en cada texto.

\section{Importancia del contexto en la comprensión lectora}

Hablar del contexto, es referirse a los elementos situacionales y condiciones ambientales que sitúa un acontecimiento. En el caso de la lectura, el contexto se entiende como la situación social, donde se conjugan los procesos comunicativos en los cuales se lee, se escribe y se habla en relación a materiales escritos (Kalman, 2003). El contexto es entendido entonces, como el espacio social donde el lector aprende (Cassany, 2010), pues tanto las acciones, las decisiones y tareas que se realizan en el momento de la lectura, y las interpretaciones que damos a los contenidos, también dependen del contexto, de las condiciones presentes en el proceso de de lectura. Se señala que la comprensión es un proceso social que se desarrolla en un ambiente determinado, donde el lector cumple con una serie de demandas y situaciones para lograr los objetivos propuestos en la lectura.

En cada espacio social donde se realiza la práctica de la lectura, el sujeto lector tiene un rol fundamental, relacionado con el cumplimiento de las tareas asignadas y responsabilidades asumidas para lograr los objetivos de lectura. Estas tareas, se orientan a una interpretación de la información, para construir el significado del texto y participar en diversas actividades de su entorno (Cassany, 2009). El texto es un instrumento de comunicación, donde el autor revela sus conocimientos y sus experiencias culturales, con el fin de compartirlas al lector. En este sentido la tarea del autor es organizar este mensaje en base a los conocimientos previos del lector, sus objetivos posibles, sus intereses, y redactar un texto de manera clara y coherente (Johnston, 1989). Así, el lector con facilidad comprenderá críticamente el discurso, se apropiará de los elementos culturales que lo componen y podrá tener una mayor aproximación a la imagen de mundo del autor.

En base a lo anterior se puede concluir que lectores y escritores establecen un puente de comunicación que les permite compartir diversas situaciones y 
visiones de mundo, dotado de voces, valores, experiencias y pensamientos que favorecen la formación cultural del sujeto. Por tanto, es preciso reflexionar sobre el carácter social de la comprensión lectora, reconociendo en ella la participación de las voces y enunciados de otros.

Cuando hablamos de comprensión lectora, nos referimos a la capacidad intelectual que posee el lector para entender textos escritos de distinta naturaleza. Se trata de apropiarse del mensaje que proporciona el texto, modificándolo, explicando su contenido, según la manera en que fue comprendido. Nos acercamos a la comprensión lectora en un sentido más profundo. Es decir, no basta con entender el código escrito Zavala (2009), o con entender las palabras de un poema, es preciso interpretar las voces que subyacen en el discurso las cuales informan de una cultura, una historia, una tradición, unos hábitos y unas prácticas comunicativas, propias de una comunidad de hablantes (Cassany 2009).

De tal manera, que "Ningún texto está solo. Cada texto se conecta con otro: utiliza palabras de otros autores" (Cassany, 2010, p. 4). Ciertamente, cada discurso se fortalece con la presencia de otras ideas, otras culturales, otras miradas, otros saberes y vivencias. Es así, que se puede afirmar que la interpretación de estas voces y puntos de vistas permitirá una mejor comprensión de los contenidos y una mejor interpretación de mundo. Queda claro, que la comprensión trasciende las líneas del texto y la mente de los seres humanos, también toma en cuenta la interacción entre las personas (Zavala, 2009). Puesto que es a través de los intercambios y convivencias en diversos espacios sociales que se producen los aprendizajes y entendimiento del medio ambiente.

\section{Conclusión}

La literatura especializada señala que el tema de la comprensión lectora, además de ser estudiado desde la concepción de la Psicología Cognitiva, también ha sido abordado desde una mirada sociocultural, y desde ya, se sustenta en diversos estudios que dan cuenta de la transformación en los modos y prácticas de la lectura y la escritura, dada su importancia dentro de la formación de todo sujeto. Una de las premisas de la teoría sociocultural es que tanto el pensamiento como el conocimiento individual son también sociales en la medida en que son producto de un intercambio de ideas, conocimiento y experiencias de distintos mundos vivido por el autor y lector.

La lectura es considerada una práctica sociocultural, al leer un texto nos encontramos con diversos elementos que dan cuenta de las posturas, de las

La comprensión lectora:

hacia una aproximación 14. sociocultural historias, de las costumbres y tradiciones que caracterizan a los hablantes de pueblos y regiones. Las prácticas culturales de unos y otros están dotadas de 
rasgos particulares muy propios de una comunidad. Tener conocimiento de ello ilumina nuestros sentidos y nos llevan a participar democráticamente en una sociedad y establecer una mejor conexión con el mundo actual.

En el proceso de la comprensión lectora es importante la responsabilidad que han de asumir tanto los escritores como los lectores. Por tanto, es preciso que el lenguaje escrito goce del uso correcto de la gramática y la fluidez del discurso, es decir el autor debe construir los significados de la manera más sencilla posible para que el lector se aproxime de manera satisfactoria a los componentes culturales presentes en el texto y se logre el intercambio social.

Finalmente se considera que el tema de la comprensión lectora ha de ser estudiado de manera consensuada entre los especialistas de la Psicología Cognitiva y la concepción sociocultural de la lectura y escritura. Con el de enriquecer los presupuestos relacionados con el tema y brindar mejores aportes al profesorado que tiene la responsabilidad de enseñar la tarea de leer, escribir y comprender.

\section{Referencias}

Ames, P. (2000). Para ser iguales, para ser distintos. Educación. Escritura y poder en Perú. Lima: IEP

Aliagas, C. (s. f.). Las prácticas lectoras adolescentes: cómo se construye el desinterés por la lectura. Universidad Pompeu Fabra, Grupo de investigación sobre Literacitat Crítica. La Rambla, 30-32. 08002-Barcelona.

Ames, P. (2000). Para ser iguales, para ser distintos. Educación. Escritura y poder en Perú. Lima: IEP

Carlino, P. (2005). Escribir, leer y aprender en la universidad. Una introducción a la alfabetización académica. Buenos Aires: Fondo de Cultura Económica.

Cassany, D. (2010). Diez claves para aprender a interpretar. Leer.es.

Cassany, D. et al. (2002). Enseñar lengua. Barcelona: Graó.

Cassany, D. (2009). Para ser letrados. Voces y miradas sobre la lectura. Barcelona: Paidós.

Cassany, D. (2010). Prácticas letradas contemporáneas: Claves para su desarrollo. <http://www.leer.es/wpcontent/uploads/webcast/documentos/ practicas_letradas/presentacion_DanielCassany.pdf $>$

Cassany, D. (2006). Tras las líneas. Sobre la lectura contemporánea. Barcelona: Anagrama.

Cassany, D y Morales, O. (2008). Leer y escribir en la universidad: Hacia la lectura y la escritura crítica de géneros científicos. Revista Memoralia (en proceso de publicación)

Contreras, R. (2008). Análisis Crítico de la Cultura. Prácticas culturales. En Contribuciones a las Ciencias Sociales. www.eumed.net/rev/cccss/0712 /rcs4.htm 
Escudero, l y León, J. (2007). Procesos inferenciales en la comprensión del discurso escrito: Influencia dela estructura deltextoenlosprocesosdecomprensión. Revista signos, 40 (64), 311-336. Recuperado de http://www.scielo.cl/ scielo. php?script=sci_arttext\&pid=S07 18-09342007000200003\&lng =es \&tlng=es. 10.4067/S0718-093420070002000 03

Escudero, I. (2010). Las inferencias en la comprensión lectora: una ventana hacia los procesos cognitivos en segunda lengua. Revista Nebrija de Lingüística Aplicada, 7 (4), 1-32.

Johnston, P. (1989). La evaluación de la comprensión lectora. Un enfoque Cognitivo. Madrid: Visor.

Kalman, J. (2003). El acceso a la cultura escrita: La participación social y la apropiación de conocimientos en eventos cotidianos de lectura y escritura. Revista Mexicana de Investigación Educativa, 3 (17), 37-66.

Kalman, J. (2000). La importancia del contexto en la alfabetización. Texto basado en la conferencia magistral presentada el 8 de septiembre en Morelia, Michoacán, México, 12-37.

Kalman, J. (2002). Saber lo que es la letra. Una experiencia de lectoescritura con mujeres de Mixquic. Instituto de la Educación de la UNESCO (1 ra. Ed.) Guadalajara: Siglo XXI.

León, J. (2001). Las inferencias en la comprensión e interpretación del discurso: Un análisis para su estudio e investigación. Revista signos, 34 (49-50), 113-125. Recuperado de http://www.scielo.cl/scielo.php?script =sci arttext\&pid=S0718-09342001004900008\&lng=es\&tIng=es. 10.4067/ S0718-09342001004900008.

León, J. y Escudero, I. (2003). Protocolos verbales en el estudio de las inferencias: Una metodología emergente. En León, J. A. (Ed.), Conocimiento y discurso. Claves para inferir y comprender (pp. 100-119). Madrid: Pirámide.

León, J.; Solari, O. y Escudero, I. (201 1). La generación de inferencias dentro de un contexto social. Un análisis de la comprensión lectora a través de protocolos verbales y una tarea de resumen oral. Revista de Investigación Educativa, 29 (1), 13-42.

Murillo, N. (2009). Tesis de Máster en Lingüística y Aplicaciones Tecnológicas. Especialidad en Aprendizaje de Lenguas. Universidad Pompeu Fabra.

Sánchez, E. (1993). Los Textos Expositivos: Estrategias para mejorar su Comprensión (2a Ed.). Salamanca: Santillana.

Zavala, V. (2009). La literacidad o lo que la gente hace con la lectura y la escritura. Textos de Didáctica de la Lengua y la Literatura, 47, 71-79. 